\title{
Multicentric reticulohistiocytosis: A case report with review
}

\author{
Vidya Kuntoji', Chandramohan Kudligi', Pradeep Vittal Bhagwat', \\ Soumyashree Krishna', Ravi M. Rathod', Arika Bansal ${ }^{2}$
}

${ }^{1}$ Department of Dermatology and Venereology and Leprosy, Karnataka Institute of Medical Sciences, Hubli, Karnataka, India, ${ }^{2}$ Consultant at Eugenix Skin and Hair Sciences, Gurgao, India

Corresponding author: Dr. Vidya Kuntoji, E-mail: drvidyakuntoji@gmail.com

\begin{abstract}
Multicentric reticulohistiocytosis also known as lipoid dermatoarthritis is a rare multisystem disorder that affects skin, mucosa and joints and has distinct histopathological features. We report a case of a 31 year old female who presented to us for joint pain involving knees and wrists and asymptomatic papulonodules over forearms knuckles and periungual region. The diagnosis of multicentric reticulohistiocytosis was made on the basis of typical histopathological features which showed multinucleated giant cells and sheets of histiocytes with abundant granular eosinophilic cytoplasm. Work up for associated diseases was negative. The patient was put on systemic steroids however was lost for follow up. The worldwide incidence of the disease is very low (around 300 cases in recent years) and it is mainly seen in Caucasians although Hispanic, African and Asian cases have also been described. Reports from India are limited and hence the present case is being reported with a brief review of the disease.
\end{abstract}

Key words: Arthritis, Histiocytosis, S100 protein

\section{INTRODUCTION}

Multicentric reticulohistiocytosis is a rare, idiopathic, multisystem disorder characterised by the association of papulonodular lesions on skin and mucous membranes along with destructive arthritis [1,2]. Also known as lipoid dermatoarthritis, the disease has the potential to involve almost any organ in the body apart from skin, bone, tendons and joints [3]. It is reported to be associated with malignancy, autoimmune diseases, hyperlipidaemia and tuberculosis [4]. It has a worldwide distribution and is seen mainly in Caucasians although Hispanic, African and Asian cases have also been described [5]. Reports from India are limited and hence the case is being reported.

\section{CASE REPORT}

A 31 year old female presented to our Dermatology Outpatient Department with complaints of joint pain, involving knees and wrists symmetrically and asymptomatic raised skin lesions since 8 months. There was associated swelling of knee joint and gradual deformity of fingers. There was no history of morning stiffness, fever, weight loss or loss of appetite. Past history of major medical and surgical illnesses and family history was insignificant. On cutaneous examination, there were multiple discrete erythematous papules and nodules of varying sizes, distributed mainly over extensor aspect of both forearms and knuckles. (Fig. 1) Similar papules were present on buccal mucosa. Periungual nodules (Fig. 2) and also a fine nodularity on ear helices were appreciated. Systemic examination was normal.

Routine blood investigations including complete hemogram, liver function tests, renal function tests and specific investigations like $\mathrm{Rh}$ factor, $\mathrm{C}$ reactive protein, ANA profile, serum lipid profile, thyroid profile was normal. Electrocardiogram, chest X-ray, USG

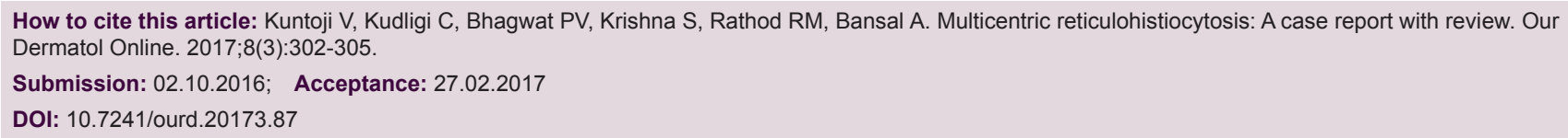


abdomen was normal. Radiograph of wrist joint and knee joint revealed minimal periarticular soft tissue swelling around wrist joint and bilateral suprapatellar bursitis. Histopathological examination revealed well

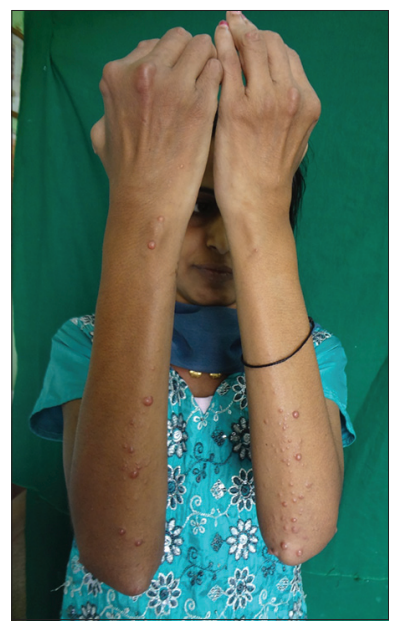

Figure 1: Multiple discrete erythematous papules and nodules over extensor aspect of both forearms.

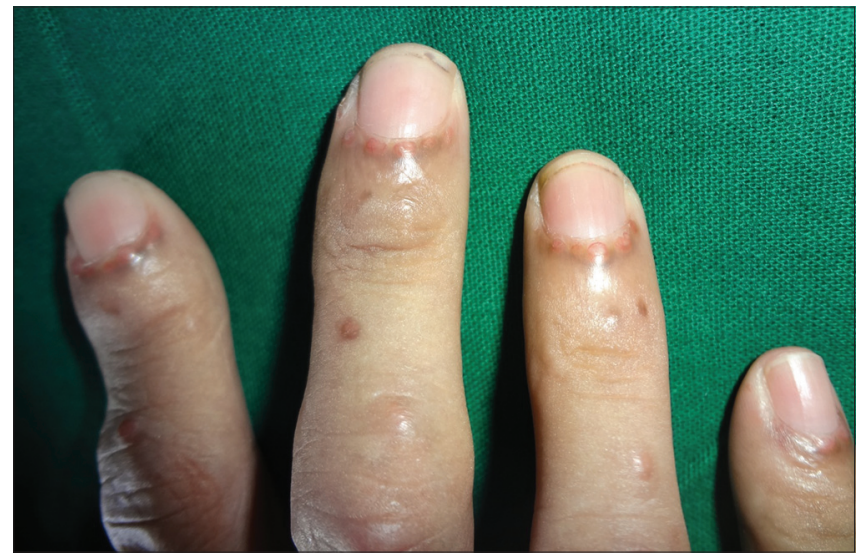

Figure 2: Periungual nodules.

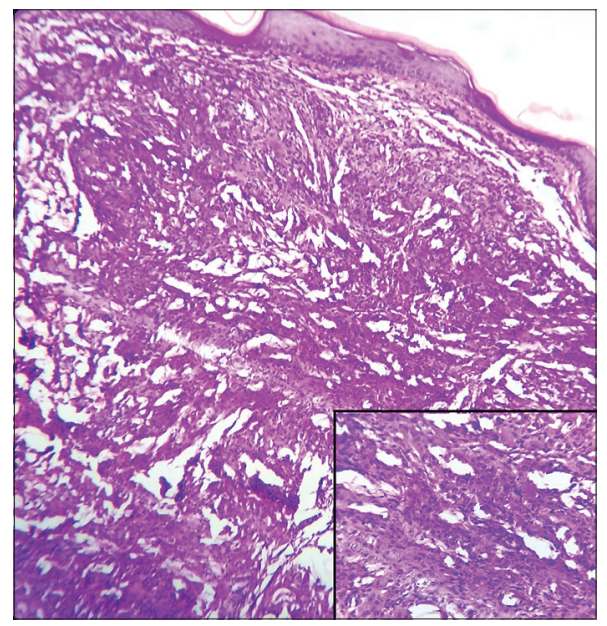

Figure 3: Biopsy from the nodule showing diffuse aggregates of histiocytes throughout the dermis. (H\&E; X10). The inset showing multinucleated giant cells (H\&E; X 40). circumscribed nodular zone comprised of sheets of histiocytes with epithelioid and xanthomatous changes in the dermis (Fig. 3). Multinucleated giant cells and histiocytes with abundant granular eosinopilic cytoplasm were appreciated which were suggestive of Multicentric reticulohistiocytosis. The patient was put on short course of systemic steroids after which the patient was lost for follow up.

\section{DISCUSSION}

Multicentric reticulohistiocytosis (MRH) is a rare non-Langerhans histiocytosis which manifests mainly with cutaneous nodules, destructive arthritis and has distinct histopathological features. It was initially described by Caro and Senear as reticulohistiocytosis granuloma in 1952 and later Goltz and Laymon coined the term multicentric reticulohistiocytosis in 1954 [6]. Although the worldwide incidence of the disease is very low, the number of cases being reported has increased from 33 in the 1954- 1969 period to around 300 cases in recent years $[5,7]$. The disease mainly affects middle aged adults and has predilection for females (female to male ratio is $3: 1$ ) [1].

The exact etiopathogenesis is not elucidated although it is believed to be due to increased levels of inflammatory cytokines like TNF-alpha, Interleukin-1, 6 and 12 by unregulated activation of macrophages. The occurrence of multinucleated giant cells in the lesions is accounted for the osteoclastic activity stimulated by Interleukin 6 [1]. While Gorman et al observed positive staining for these cytokines on immunohistochemical analysis of synovial tissue; Bennassar et al reported increased serum cytokine levels which decreased after treatment $[8,9]$.

The cardinal symptoms include those of joint and skin diseases. While inflammatory joint disease is a frequent presenting symptom, and is sole symptom in $45 \%$ of patients, skin disease is the presenting manifestation in $30 \%$ of patients [10]. Arthropathy usually precedes skin lesions (50\%); skin lesions may precede disease $(25 \%)$ or both can occur concurrently (25\%) [3]. It manifests with diffuse, symmetric, progressive and destructive polyarthritis affecting interphalangeal joints mainly the distal interphalangeal joints (75\%) and other joints like knees, wrists, shoulders and elbows. It may progress to debilitating arthritis mutilans $[2,5]$. Cutaneous lesions are usually multiple erythematous to brown papulonodules which may coalesce to plaques mainly seen over ears, perinasal region, dorsal aspect 
of hands and trunk. The appearance of papules in the periungual region is described as "coral beads" [5]. Involvement of oral and nasal mucosa is seen in roughly one-third of the cases [3]. Constitutional symptoms like fever, malaise, weakness, weight loss, anorexia may be present and the disease can involve multiple organ systems including cardiac and skeletal muscle, the pleura and gastrointestinal tract $[4,11]$. Our patient did not have mucosal involvement, constitutional symptoms and imaging studies did not show internal organ involvement.

The clinical features can sometimes lead to misleading diagnosis as they closely mimic other diseases like dermatomyositis, rheumatoid arthritis. Cheng $\mathrm{L}$ and Chiang Y [5] reported a case of MRH in association with Sjogren syndrome in addition to skin lesions mimicking Gottron's papules and periungual erythema. Fett N et al noted 27 patients with dermatomyositis like features in a systematic review on 234 cases of MRH [12]. Millar A et al reported a referred suspected case of weakly RF-positive Rheumatoid arthritis with skin nodules which proved to be MRH on histopathology and further investigations showed its association with metastatic large cell carcinoma [13]. This implies that the disease may be often underdiagnosed because of its rarity. The other differential diagnosis includes lepromatous leprosy, sarcoidosis, xanthomatosis, histiocytosis X, juvenile and adult xanthogranuloma, generalized eruptive histiocytoma, familial histiocytic dermatoarthritis and neurofibromatosis [14]. The cutaneous manifestations with erosive arthritis differentiates MRH from some of these conditions.

There is no direct evidence of involvement of any infectious organism in its etiopathogenesis. However, there is evidence of tuberculosis exposure in one third of cases with active tuberculosis present in $5 \%$ [11]. Hyperlipidaemia is seen in 10-20\% cases. It is associated with autoimmune diseases in $5-20 \%$ cases [5]. These include Sjogren's syndrome, primary biliary cirrhosis, systemic sclerosis, SLE, celiac disease, myopathy, diabetes mellitus and hypothyroidism $[4,5]$. Although there is varied school of thoughts regarding paraneoplastic phenomena [15] associations have been made with malignancies (approximately $25-30 \%$ cases) like carcinoma of breast, cervix, ovary, stomach, colon, lung and bronchus, melanoma, lymphoma, leukaemia and sarcoma [14]. These associations underpin the vital role of screening for these diseases before starting treatment.
Laboratory investigations may reveal nonspecific findings like microcytic anaemia, elevated ESR, CRP and hyperlipidaemia. Radiologic findings include erosions which are initially circumscribed and later spread from margin to involve entire joint surface, widening of joint spaces, loss of cartilage and resorption of subchondral bone. Involvement of interphalangeal joints of hands can lead to "opera glass" hand. These findings help in arriving at a diagnosis; albeit other forms of erosive polyarthritis like rheumatoid arthritis, psoriatic arthritis, and erosive osteoarthritis should be meticulously ruled out $[2,14]$. Our patient did not have any of the above mentioned abnormal lab parameters or imaging features except for suprapatellar bursitis. Histological and immunological features form the main crux in confirmation of the diagnosis of this condition. The finding of dermal infiltration of mononuclear and multinucleated giant cells with eosinophilic "ground glass" PAS positive cytoplasm is typical of MRH [15]. These cells are positive for TRAP, CD68, lysozyme and human alveolar macrophage-56 (HAM-56) whereas negative for S100protein, CDla and factor XIIIa [3]. However it is noteworthy to mention that Bialy Nicki-B R et al [7] had observed S100 positivity. The histological features of the nodules seen in our patient were typical of MRH; however due to financial constraints, immunohistochemistry was not done.

The prognosis and clinical course is unpredictable and the disease may remit spontaneously. However, without treatment it will lead to debilitating arthropathy and cutaneous scarring [4]. There are no guidelines per se for its treatment. Non-steroidal anti-inflammatory drugs and corticosteroids are the preferred drugs in the initial line of management. They usually alleviate pain and are not much helpful for remissions. Other treatment modalities used with some success include Methotrexate, Mycophenolate Mofetil, azathioprine, cyclophosphamide, cyclosporine, chlorambucil [3,5]. Improvement has also been noted with Biphosphonates like alendronate and zolidronate [2]. As per one consensus, systemic steroid plus cyclophosphamide or chlorambucil should be used for skin involvement and systemic steroid plus methotrexate should be considered for joint involvement [4]. Pacheco-Tena C et al reported Tocilizumab could be an alternative for refractory cases of MRH [16].

Recent developments focus on TNF antagonists which are said to induce remission and also allow a reduction in steroid dosage in refractory cases of MRH. As per the systematic review made between January 2003 and 
April 2014 by Zhao $\mathrm{H}$ et al., a total of 17 cases were found who were treated with anti- TNF-alpha agents like Etanercept, Infliximab, and Adalimumab with or without concomitant therapies mainly systemic steroids, Methotrexate, Hydroxychloroquine, and Mycophenolate Mofetil. Majority had improvement in skin and joint symptoms with very few patients experiencing fever, weight loss, fatigue, night sweats, stiffness and muscle aches [17]. Local treatment of the visibly perturbing lesions has also been described. Mahajan RS et al reported a case of MRH with cosmetically disfiguring cutaneous lesions which were successfully treated with CO2 laser [18]. All these reports suggest that the treatment of MRH is case based.

\section{CONCLUSION}

MRH is a rare and often underdiagnosed condition. Dermatologists and physicians should be aware of its closely mimicking presentations with other diseases. Due to its implicated association with malignancies and autoimmune diseases, early and aggressive treatment and regular follow up of the patient is of paramount importance.

\section{REFERENCES}

1. Tran TH, Pope E, Weitzman S. Cutaneous histiocytoses. In: Griffiths CEM, Barker J, Bleiker T, Chalmers R, Creamer D, editors. Rook's Text book of Dermatology. 9th ed. Oxford: Blackwell science; p.136.22-136.23.

2. Saba R, Kwatra SG, Upadhyay B, Mirrakhimov AE, Khan FN. Multicentric Reticulohistiocytosis Presenting with Papulonodular Skin Lesions and Arthritis Mutilans. Case Rep Rheumatol. 2013;2013:1-4

3. Shah SP, Shah AM, Prajapati SM, Bilimoria FE. Multicentric reticulohistiocytosis. Indian Dermatol Online J. 2011;2:85-7.

4. Koh CC, Jong KK. Reticulohistiocytosis: a case series in Hong Kong. Hong Kong J Dermatol Venereol. 2012;20:5-11.
5. Cheng L, Chiang Y. Multicentric reticulohistiocytosis in a Taiwanese woman with Sjogren syndrome. Dermatol Sinica. 2016;42-5.

6. Rezaieyazdi Z, Sandooghi M, Torghabe HM, Derhami A. Multicentric reticulohistiocytosis: A case report. Acta Med Iran. 2005;43:372-6.

7. Bialynicki-Birula R, Sebastian-Rusin A, Maj J, Wozniak Z, Baran E, Dziegiel P. Multicentric Reticulohistiocytosis with S100 Protein Positive Staining: A Case Report. Acta Dermatovenerol Croat. 2010;18:35-7.

8. Gorman JD, Danning C. Schumacher HR, Klippel JH, Davis JC. Multicentric reticulohistiocytosis: Case report with immunohistochemical analysis and literature review. Arthritis Rheum. 2000:930-8.

9. Bennassar A, Mas A, Guilabert A, Julia M, Mascaro-Galy JM, Herrero C. Multicentric reticulohistiocytosis with elevated cytokine serum levels. J Dermatol. 2011;905-10.

10. Haridas V, Haridas K. Multicentric Reticulohistiocytosis. J Case Rep. 2015;5:160-2.

11. Campbell DA, Edwards NL. Multicentric reticulohistiocytosis: systemic macrophage disorder. Bailliers Clin Rheumatol. 1991;5:301-19.

12. Fett N, Liu RH. Multicentric reticulohistiocytosis with dermatomyositis-like features: a more common disease presentation than previously thought. Dermatology. 2011;222:1002-8.

13. Millar A, Kane DO, Taggart A. Multicentric reticulohistiocytosis: a lesson in screening for malignancy. Rheumatology. 2008;47:1103-4.

14. Yap FB. Multicentric reticulohistiocytosis in a Malaysian Chinese lady: A case report and review of literature. Dermatol Online J. 2009;15:2

15. Santilli D, Monaco AL, Cavazzini PL, Trotta F. Multicentric reticulohistiocytosis: a rare cause of erosive arthropathy of the distal interphalangeal finger joints. Ann Rheum Dis. 2002;61:485-7.

16. Tena C, Rees-Codero G, Ochoa-Albiztegui R, Rios-Barrera V, Gonzalez-Chavez SA. Treatment of multicentric reticulohistiocytosis with toclizumab. J Clin Rheumatol. 2013;19:272-6.

17. Zhao H, Chunmei WU, Mengyun WU, Zhou Y, Zhu H, Li Y, et al. Tumor Necrosis Factor Antagonists In The Treatment Of Multicentric Reticulohistiocytosis: Current Clinical Evidence. Mol Med Rep. 2016;14:209-17.

18. Mahajan RS, Shah AC, Nagar A, Freny BE. Treatment of facial lesions of multicentric reticulohistiocytosis by carbon dioxide laser. J Cutan Aesthet Surg. 2013;6:161-3.

Copyright by Vidya Kuntoji, et al. This is an open-access article distributed under the terms of the Creative Commons Attribution License, which permits unrestricted use, distribution, and reproduction in any medium, provided the original author and source are credited.

Source of Support: Nil, Conflict of Interest: None declared. 